\title{
Generalization of the ERIT Principle and Method
}

\author{
A. Ruggiero
}

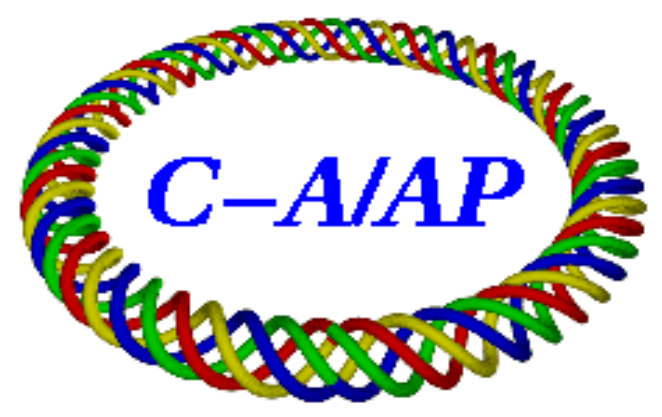

Collider-Accelerator Department Brookhaven National Laboratory Upton, NY 11973

Notice: This document has been authorized by employees of Brookhaven Science Associates, LLC under Contract No. DE-AC02-98CH10886 with the U.S. Department of Energy. The United States Government retains a nonexclusive, paid-up, irrevocable, world-wide license to publish or reproduce the published form of this document, or allow others to do so, for United States Government purposes. 


\title{
Generalization of the ERIT Principle and Method
}

\author{
Alessandro G. Ruggiero \\ Brookhaven National Laboratory, PO Box 5000, Upton, Long Island, NY 11973, USA
}

\begin{abstract}
The paper describes the generalization of the method to produce secondary particles with a low-energy and low-intensity primary beam circulating in a Storage Ring with the Emittance-Recovery by Internal-Target (ERIT).
\end{abstract}

Keywords: Storage Ring; Ionization Cooling: Production of Secondary Beams; FFAG; ERIT.

\section{A BEAM OF IONS CIRCULATING IN A STORAGE RING}

A beam of ions completely stripped of their electrons circulates in a Storage Ring of circumference C. The central beam kinetic energy is $\mathrm{E}$ and the spread (rms value) $\sigma_{\mathrm{E}}$. There is no net acceleration though the beam is bunched by a constant frequency RF cavity system in the several MHz range. There is only one bunch so that the revolution frequency $f_{0}=\beta c / \mathrm{C}$ equals the RF frequency $f_{\mathrm{RF}}$. We denote with $\beta$ and $\gamma$ the usual velocity and energy relativistic factors, and with $c$ the speed of light. Also $p$ is the beam central momentum, and B $\rho$ the corresponding value of the magnetic rigidity. The mass number of the ion is $\mathrm{A}$, and the charge state equals the atomic number $\mathrm{Z}$. There are $\mathrm{N}$ ions circulating at any time. Denoting with $e$ the elementary electric charge, the average particle -current is

$$
\mathrm{I} \quad \mathrm{N} e f_{0}
$$

There is a thin Foil inserted at one location of the ring that is traversed by the beam of ions periodically, every turn. The foil is made of stationary, solid material made of atoms of mass number $\mathrm{A}_{\mathrm{F}}$ and atomic number $\mathrm{Z}_{\mathrm{F}}$. Of course the atoms are neutral and are surrounded by a corresponding number $\left(\mathrm{Z}_{\mathrm{F}}\right)$ of electrons. Let us denote with $\delta$ the mass density of the Foil (in $\mathrm{g} / \mathrm{cm}^{3}$ ) and with $\rho$ the atom density (in $\mathrm{cm}^{-3}$ )

$$
\rho \quad=\quad \delta / \mathrm{A}_{\mathrm{F}} m_{\mathrm{p}}
$$

where $m_{\mathrm{p}}=1.67 \times 10^{-24} \mathrm{~g}$ is the proton mass at rest. The Foil (Target) has a thickness $g$ and a transverse extension wide enough to intercept the whole beam at every traversal.

When an ion of the circulating beam traverses the Foil, one of the following events will occur:

1. The ion trajectory widens transversally by Multiple Coulomb Scattering (MCS);

2. There is a net Energy Loss (EL) of the same value to all ions;

3. There is a widening of the energy spread (Energy Straggling (ES));

4. The ion scatters with a nucleus triggering a nuclear reaction;

5. Single Coulomb Scattering (SCS) at large angle and large energy that in one event removes the particle from the aperture of the Storage Ring.

Events 1, 2, and 3 cause a continuous, stochastic increase of the beam dimensions and spreads, but not beam loss, unless the growth after many turns is unchecked, and the edge of the beam reaches the available aperture of the Storage Ring. These events together are described by a total cross-section $\sigma_{\mathrm{T}}$ that is very large, ranging in the hundred of barns (one barn $=10^{-24} \mathrm{~cm}^{-2}$ ). On the other side, Event 4 causes immediately the loss of the particle, but it is also the useful event, as the resulting nuclear reaction is the one sought by the set-up. Typically the cross-section 
$\sigma_{\mathrm{N}}$ of this event is in the barn range, and thus much smaller than $\sigma_{\mathrm{T}}$. Event 5 needs of course to be estimated and included in the analysis, but it is usually of no consequences to the beam performance, and to the operation of the set-up, as long as the Storage Ring has sufficiently large aperture, and the beam loss can be compensated continuously by an adequate ion source ${ }^{*}$. There are other effects that may cause disruption of the beam operation and performance, like, for example, the residual pressure in the vacuum chamber of the Storage Ring, magnet power supply stability, and resonances intrinsic to the Ring Lattice. But these effects are independent of the presence of the Foil, can be in principle controlled and compensated for. We shall assume that this is indeed the case.

\section{PRODUCTION OF SECONDARY PARTICLES BY NUCLEAR REACTIONS}

The nuclear reaction that is sought with Event 4 listed above can symbolically described by

$$
\mathrm{A}+\mathrm{A} F \quad \rightarrow \quad \mathrm{B}+\mathrm{X}
$$

where $\mathrm{A}$ is the ion circulating in the Storage Ring colliding with ions $\mathrm{A}_{\mathrm{F}}$ in the Foil, $\mathrm{B}$ is the by-product, and $\mathrm{X}$ the element that is actually sought in the production. Among others, for instance, the following nuclear reactions are of interest for several applications that include neutron and gamma-rays production, nuclear energy production by fusion reaction, and more:

$$
\begin{array}{lllll}
\mathrm{p} & +{ }^{7} \mathrm{Li} & \rightarrow & { }^{7} \mathrm{Be}+\mathrm{n} \\
\mathrm{p}+{ }^{9} \mathrm{Be} & \rightarrow & { }^{9} \mathrm{~B}+\mathrm{n} \\
\mathrm{p}+{ }^{13} \mathrm{C} & \rightarrow & { }^{14} \mathrm{~N}+\gamma(7.55 \mathrm{MeV}) \\
\mathrm{p}+{ }^{11} \mathrm{~B} & \rightarrow & 3 \alpha+8.7 \mathrm{MeV} \\
\mathrm{d}+\mathrm{T} & \rightarrow & \alpha+\mathrm{n}(17.6 \mathrm{MeV})
\end{array}
$$

When this method of secondary beam production is proposed with the primary beam circulating in a Storage Ring, it is usually criticized by the fact that the total cross-section $\sigma_{\mathrm{T}}$ is much larger than the desired nuclear reaction cross-section $\sigma_{\mathrm{N}}$. The perception is that the primary beam is completed depleted before it could be effectively used. In reality, as we already noted, the total cross-section does not cause immediate beam loss, but only an enlargement of the beam dimensions and spreads. In fact the growth can be controlled with some of the cooling techniques available, namely: Ionization, Sympathetic, and Electron Cooling ${ }^{* *}$. The cooling is needed to counteract the growth caused by the total cross-section and to maintain the beam at constant dimensions and confined in the Storage Ring only for a required period of time.

The method of Emittance-Recovery by Internal-Target (ERIT) was proposed by Y. Mori and others [1] for the production of neutrons according to the second nuclear reaction (5) listed above. The layout of the ERIT project being assembled at KURRI (Kyoto University Research Reactor Institute) is shown in Figure 1. Its main purpose is the production of neutrons for Boron Neutron Capture Therapy (BNCT) of cancer. It makes use of the Ionization Cooling, with the Foil-Target itself used as the ionization media. This method can be generalized and applied to all other types of nuclear reactions. Of course other Cooling Techniques, like Electron and Sympathetic Cooling, can be used in the place of Ionization Cooling.

\section{PRODUCTION RATES}

The local rate of events leading to the production of the element $\mathrm{X}$ per incident ion is

$$
\mathrm{d} n / \mathrm{dt}=\beta c \rho \sigma_{\mathrm{N}}
$$

To get the average rate of production we need to multiply this by the ratio g / C of the Foil thickness to the Ring circumference

$$
\mathrm{d}\left\langle n>/ \mathrm{dt}=\beta c \rho g \sigma_{\mathrm{N}} / \mathrm{C}=f_{0} \rho g \sigma_{\mathrm{N}}\right.
$$

\footnotetext{
* There are of course possibilities of beam loss also through other non-useful nuclear reactions that need to be evaluated for each case. See comments regarding this phenomenon at the end of the report.

${ }^{* * *}$ There is of course also Laser Cooling, that though requires the ions to be only partially stripped, since few orbiting electrons are needed for the laser excitation. We shall not consider this case here.
} 
from which we derive the average time $\tau$ the ion spends circulating from injection to the instant it is removed by the nuclear scattering event

$$
\tau \quad=\quad 1 / f_{0} \rho g \sigma_{\mathrm{N}}
$$

or the equivalent expected number of revolutions

$$
n_{\mathrm{rev}} \quad=\quad \tau f_{0} \quad=\quad 1 / \rho g \sigma_{\mathrm{N}}
$$

and the free path length within the Foil

$$
\lambda \quad=\quad n_{\text {rev }} g=1 / \rho \sigma_{\mathrm{N}}
$$

Since we assume the beam is injected with reasonable small dimensions and spreads, all particles spend in average a time $\tau$ circulating in the Storage Ring, the beam size growth needs only to be estimated as occurring over the same period of time. The growth beyond that has no consequences to the beam as particles are continuously removed by the single events of nuclear scattering. At the same time, to maintain the beam intensity at the constant value, the beam is to be continuously replenished by the ion source placed outside the Ring. The total production rate of the element $\mathrm{X}$ is thus

$$
\mathrm{d} n_{\mathrm{tot}} / \mathrm{dt}=\mathrm{Nd}<n>/ \mathrm{dt}=\mathrm{I} \rho g \sigma_{\mathrm{N}} / e
$$

which is the main requirement of the set-up. The requirement on the ion source for continuous top-off is then

$$
\mathrm{I}_{\mathrm{s}} \quad=\quad e \mathrm{~d} n_{\mathrm{tot}} / \mathrm{dt}=\operatorname{I\rho g} \sigma_{\mathrm{N}}
$$

The operation duty cycle

$$
\eta \quad=\quad \mathrm{I}_{\mathrm{S}} / \mathrm{I}=\rho g \sigma_{\mathrm{N}}
$$

which, for the system to be efficient, should be kept as small as possible.

\section{DISCUSSION}

For a given nuclear reaction most of the parameters are assigned, like the beam energy E, the cross-section $\sigma_{\mathrm{N}}$, and the Foil material density $\rho$. What is left undefined is the total production rate $\mathrm{d} n_{\text {tot }} / \mathrm{dt}$, the ion survival time $\tau$, the total number of ions N, the Ring circumference C, and the Foil thickness $g$. It is seen that the following simple relation holds

$$
\mathrm{d} n_{\text {tot }} / \mathrm{dt} \quad=\quad \mathrm{N} / \tau
$$

Since $\mathrm{d} n_{\text {tot }} / \mathrm{dt}$ is a requirement, the ratio $\mathrm{N} / \tau$ is an invariant. For the beam economy, one may prefer a lower intensity $\mathrm{N}$ and, thus, a smaller value of $\tau$. This is a favorable condition because a smaller value of $\tau$ gives the beam a better chance to survive the growth from MCS, the energy loss and straggling. At the same time a smaller value of $\tau$ requires a large ratio $g / \mathrm{C}$, that is a long Target and a small Ring circumference (compatible with the beam energy). Also, for a given Target thickness $g$, the average production rate is proportional to the average line density $\mathrm{N} / \mathrm{C}$. A lower beam intensity $\mathrm{N}$ corresponds to a smaller Ring circumference (again, as long as it is compatible with the beam energy).

\section{EXAMPLES}

In the following we shall consider three examples that have been investigated in more details The examples correspond to the nuclear reactions (5), (6) and (7) listed above. The main parameters are given in Table 1. 
TABLE 1. Main Parameters of Three Nuclear Reactions for Secondary Particle Production.

\begin{tabular}{llll}
\hline & $\mathbf{p}+{ }^{\mathbf{9}} \mathbf{B e}->{ }^{\mathbf{9}} \mathbf{B}+\mathbf{n}$ & $\mathbf{p}+{ }^{\mathbf{1 3}} \mathbf{C}->{ }^{14} \mathbf{N}+\gamma$ & Boron \\
\hline Target Material & Beryllium & Carbon $->\mathbf{3} \alpha$ \\
Mass Number $\mathrm{A}_{\mathrm{F}}$ & 9 & 13 & 11 \\
Atomic Number $\mathrm{F}_{\mathrm{F}}$ & 4 & 6 & 5 \\
Mass Density, $\delta$ & $1.85 \mathrm{~g} / \mathrm{cm}^{3}$ & $2.27 \mathrm{~g} / \mathrm{cm}^{3}$ & $2.37 \mathrm{~g} / \mathrm{cm}^{3}$ \\
Atomic Density, $\rho$ & $1.23 \times 10^{23} / \mathrm{cm}^{3}$ & $1.05 \times 10^{23} / \mathrm{cm}^{3}$ & $1.29 \times 10^{23} / \mathrm{cm}^{3}$ \\
Primary Beam & Proton & Proton & Proton \\
Mass Number A & 1 & 1 & 1 \\
Atomic Number $\mathrm{Z}$ & 1 & 1 & 1 \\
Kinetic Energy, E & $10 \mathrm{MeV}$ & $1.8 \mathrm{MeV}$ & $0.75 \mathrm{MeV}$ \\
$\beta$ & 0.14484 & 0.06185 & 0.0400 \\
Magnetic Rigidity, B $\rho$ & $4.5815 \mathrm{kGauss}-\mathrm{m}$ & $1.9395 \mathrm{kGauss}-\mathrm{m}$ & 1.2516 \\
Circumference, $\mathrm{C}$ & $9.60 \mathrm{~m}$ & $4.06 \mathrm{~m}$ & $2.62 \mathrm{~m}$ \\
Revolution Frequency, $f_{0}$ & $4.53 \mathrm{MHz}$ & $4.56 \mathrm{MHz}$ & $4.57 \mathrm{MHz}$ \\
Circulating Current, I & $7.24 \mathrm{~mA}$ & $7.30 \mathrm{~mA}$ & $7.31 \mathrm{~mA}$ \\
Secondary Particle & neutron $(\sim \mathrm{MeV})$ & gamma $(7.55 \mathrm{MeV})$ & $3 \mathrm{alpha}(8.75 \mathrm{MeV})$ \\
Cross-Section, $\sigma_{\mathrm{N}}$ & $500 \mathrm{mbarn}$ & $10 \mathrm{mbarn}$ & $1100 \mathrm{mbarn}$ \\
Production Rate, $\mathrm{d} n_{\text {tot }} / \mathrm{dt}$ & $2.8 \times 10^{12} / \mathrm{s}$ & $4.8 \times 10^{10} / \mathrm{s}$ & $6.5 \times 10^{12} / \mathrm{s}$ \\
Survival Time, $\tau$ & $3.6 \mathrm{~ms}$ & $210 \mathrm{~ms}$ & $1.54 \mathrm{~ms}$ \\
Free Path Length, $\lambda$ & $16.2 \mathrm{~cm}$ & $956 \mathrm{~cm}$ & $7.05 \mathrm{~cm}$ \\
Number of Turns, $n_{\text {rev }}$ & $16.2 \times 10^{3}$ & $0.96 \times 10^{6}$ & $7.05 \times 10^{3}$ \\
Duty Factor, $\eta$ & $6.2 \times 10^{-5}$ & $1.0 \times 10^{-6}$ & $1.4 \times 10^{-4}$ \\
Ion Source Current, $\mathrm{I}_{\mathrm{s}}$ & $0.446 \mu \mathrm{A}$ & $0.0076 \mu \mathrm{A}$ & $1.038 \mu \mathrm{A}$ \\
\hline
\end{tabular}

Table 1 shows also the circumference $\mathrm{C}$ of the corresponding Storage Ring, and the revolution frequency $f_{0}$ assuming, as an example, a bending field of $10 \mathrm{kGauss}$ and a bending magnet packing factor of $30 \%$. Other quantities are also shown, e.g. the total production rate $\mathrm{d} n_{\mathrm{tot}} / \mathrm{dt}$, the survival time $\tau$, the free path length $\lambda$, the number of revolutions $n_{\text {rev }}$, and the duty factor $\eta$. For the estimate of these quantities, we have taken, quite arbitrary, the total number of circulating protons $\mathrm{N}=10^{10}$ and the thickness of the Foil $g=10 \mu \mathrm{m}$, from which other values can be derived by proper (linear and inverse) scaling.

The parameters of Table 1 are very reasonable, and highly desirable for the production of the secondary particles. But, as the primary beam crosses the Foil, other effects take over that spoil the beam stability. These effects also are described by their own characteristic times that are to be compared to the survival time $\tau$.

\section{MULTIPLE COULOMB SCATTERING}

Multiple Coulomb Scattering (MCS) causes the beam to diffuse laterally according to the equations

$$
\mathrm{d} \theta_{0}^{2} / \mathrm{ds}=0.157 \mathrm{Z}_{\mathrm{F}}\left(\mathrm{Z}_{\mathrm{F}}+1\right) \mathrm{Z}^{2} \mathrm{~B}_{\mathrm{M}} / \mathrm{A}_{\mathrm{F}}(p c)^{2} \beta^{2}
$$

which is the growth of the rms scattering angle (in radians) per unit of path length (in $\mathrm{g} / \mathrm{cm}^{2}$ ). The momentum $p$ is in $\mathrm{MeV} / \mathrm{c}$, and $\mathrm{B}_{\mathrm{M}}$ a coefficient derived from the Moliere's theory of MCS [2]. The actual beam emittance growth, in either pane, is measured by $\varepsilon=\beta_{\mathrm{L}} \theta_{0}^{2} / 2$ where $\beta_{\mathrm{L}}$ is the betatron function at the Foil location that we assume to have the same value in both planes. The average emittance growth per unit of time is

$$
\begin{aligned}
\mathrm{d} \varepsilon / \mathrm{dt} & =0.157 \mathrm{Z}_{\mathrm{F}}\left(\mathrm{Z}_{\mathrm{F}}+1\right) \mathrm{Z}^{2} g c \delta \mathrm{B}_{\mathrm{M}} \beta_{\mathrm{L}} / 2 \mathrm{~A}_{\mathrm{F}} \mathrm{C}(p c)^{2} \beta \\
& =\mathrm{D}_{\mathrm{T}}
\end{aligned}
$$

In the following we shall take $\beta_{\mathrm{L}}=1 \mathrm{~m}$ for all cases, and continue to assume $g=10 \mu \mathrm{m}$. The amount of emittance growth $\Delta \varepsilon$ after the survival time $\tau$ is given by $\tau \mathrm{d} \varepsilon / \mathrm{dt}$ and is independent of the foil thickness, though the Moliere's coefficient has a somewhat weak dependence on $g$. The results are shown in Table 2 . The final emittance $\Delta \varepsilon$ is too large to be contained by a reasonable physical aperture of the Storage Ring in all three cases considered. 
TABLE 2. Multiple Coulomb Scattering. Energy Loss. Energy Struggling.

\begin{tabular}{llll}
\hline & $\mathbf{p}+{ }^{9} \mathbf{B e}->{ }^{9} \mathbf{B}+\mathbf{n}$ & $\mathbf{p}+{ }^{13} \mathbf{C}->{ }^{\mathbf{1 4}} \mathbf{N}+\gamma$ & p $+{ }^{\mathbf{1 1}} \mathbf{B}->\mathbf{3} \alpha$ \\
\hline Target Material & Beryllium & Carbon & 11 \\
Moliere's Coefficient, $\mathrm{B}_{\mathrm{M}}$ & 8 & 10 & $3,611 \mathrm{p} \mathrm{m}-\mathrm{rad} / \mathrm{s}$ \\
MCS Growth Rate, $\mathrm{d} \varepsilon / \mathrm{dt}$ & $9.4 \mathrm{p} \mathrm{m-rad} / \mathrm{s}$ & $647 \mathrm{p} \mathrm{m}-\mathrm{rad} / \mathrm{s}$ & $5.57 \mathrm{p} \mathrm{m}-\mathrm{rad}$ \\
Emittance Growth, $\Delta \varepsilon$ & $0.0337 \mathrm{p} \mathrm{m}-\mathrm{rad}$ & $135 \mathrm{p} \mathrm{m}-\mathrm{rad}$ & $280 \mathrm{MeV} /\left(\mathrm{g} / \mathrm{cm}^{2}\right)$ \\
Stopping Power, $\mathrm{S}$ & $38 \mathrm{MeV} /\left(\mathrm{g} / \mathrm{cm}^{2}\right)$ & $154 \mathrm{MeV} /\left(\mathrm{g} / \mathrm{cm}^{2}\right)$ & $3,030 \mathrm{GeV} / \mathrm{s}$ \\
Energy Loss Rate, dE/dt & $318 \mathrm{GeV} / \mathrm{s}$ & $1,600 \mathrm{GeV} / \mathrm{s}$ & $4.68 \mathrm{GeV}$ \\
Total Energy Loss, $\Delta \mathrm{E}$ & $1.14 \mathrm{GeV}$ & $334 \mathrm{GeV}$ & $660 \mathrm{keV}$ \\
Energy Loss / Revolution & $70 \mathrm{keV}$ & $350 \mathrm{keV}$ & $767 \mathrm{MeV}^{2} / \mathrm{s}$ \\
Energy Diffusion, $\mathrm{d} \sigma_{\mathrm{E}}{ }^{2} / \mathrm{dt}$ & $585 \mathrm{MeV} / \mathrm{s}$ & $746 \mathrm{MeV}^{2} / \mathrm{s}$ & $1.2 \mathrm{MeV}^{2}$ \\
Spread Growth, $\Delta \sigma_{\mathrm{E}}{ }^{2}$ & $2.1 \mathrm{MeV}^{2}$ & $156 \mathrm{MeV}^{2}$ & \\
\hline
\end{tabular}

\section{AVERAGE ENERGY LOSS}

Let us turn now our attention to the average rate of energy loss when crossing the Foil. This is given by the Stopping Power equation [2]

$$
\begin{array}{rll}
\mathrm{S} & =\left(0.30708 / \beta^{2}\right) \mathrm{Z}^{2}\left(\mathrm{Z}_{\mathrm{F}} / \mathrm{A}_{\mathrm{F}}\right)\left[\ln \left(2 m c^{2} \beta^{2} \gamma^{2} / \mathrm{I}_{\mathrm{AV}}\right)-\beta^{2}\right] \\
& =-\mathrm{dE} / \mathrm{ds}
\end{array}
$$

where the electron shell correction and the density correction factor at high energies have been neglected. In this equation $m$ is the mass at rest of the electron, and $\mathrm{I}_{\mathrm{AV}}$ is the mean excitation energy, a function of the Target atomic number $\mathrm{Z}_{\mathrm{F}}$. Values of the Stopping Power $\mathrm{S}$ for actual cases can be read from available Tables [2], and they are listed in Table 2. The actual average rate of energy loss per unit of time is

$$
\mathrm{dE} / \mathrm{dt}=\delta \beta c \mathrm{Sg} / \mathrm{C}
$$

which is proportional to the Foil thickness $g$. The average amount of energy lost during the survival time $\tau$ is then $\Delta \mathrm{E}=\tau \mathrm{dE} / \mathrm{dt}$, independent of the Foil thickness. All these quantities are shown in Table 2 for the cases under consideration. The total energy lost $\Delta \mathrm{E}$ is a too large quantity that if not compensated will cause the beam to be lost after few revolutions. There is thus need to continuously re-accelerate the beam every turn as it loses energy to the Foil. We show in Table 2 also the energy lost per turn $\mathrm{dE} / \mathrm{dn}=\left(1 / f_{0}\right) \mathrm{dE} / \mathrm{dt}$ which needs to be re-supplied back to the beam by an RF cavity system placed in another location of the Storage Ring. Of course the RF peak voltage ought to be large enough to accommodate the actual beam bunch area. The amount of RF voltage required seems to be acceptable and feasible for all the cases considered.

\section{ENERGY STRUGGLING}

The scattering of the ions with the electron clouds surrounding the nuclei of the Foil not only causes an average energy loss, but also an increase of the energy distribution spread [2] at the rate given by, in average,

$$
\mathrm{d} \sigma_{\mathrm{E}}^{2} / \mathrm{dx} \quad=\quad\left(26 \times 10^{-26} \mathrm{MeV}^{2} / \mathrm{cm}\right) \mathrm{Z}_{\mathrm{F}} \gamma^{2}\left(1-\beta^{2} / 2\right) \rho g / \mathrm{C}
$$

where the atom density $\rho$ is to be expressed in $\mathrm{cm}^{-3}$ and the path length $\mathrm{x}$ in $\mathrm{cm}$. From Eq. (22) we derive the energy diffusion rate $\mathrm{D}_{\mathrm{L}}=\mathrm{d} \sigma_{\mathrm{E}}^{2} / \mathrm{dt}=\beta c \mathrm{~d} \sigma_{\mathrm{E}}^{2} / \mathrm{dx}$. The maximum expected rms growth after the survival time $\tau$ is

$$
\Delta \sigma_{\mathrm{E}}^{2}=\quad \tau \mathrm{D}_{\mathrm{L}}
$$

In conclusion, if a proton is let to circulate for a period of time corresponding to $\tau$, chances are it would not survive, in all the cases considered, any of the effects from MCS, Energy Loss, and Energy Straggling. To reduce the magnitude of these effects it may be possible to shorten the beam circulation period in the Storage Ring to a fraction of the survival time $\tau$. But even in this case the most serious effect is the energy loss (caused by the atomic 
electrons) that needs to be compensated by re-accelerating the beam. The re-acceleration though introduces damping of the transverse oscillations but, unfortunately, also anti-damping of the longitudinal oscillations. This is called Ionization Cooling.

\section{IONIZATION COOLING}

The energy loss of the proton traversing the Foil is accompanied by a momentum reduction that is directed primarily along the main direction of motion that makes an angle with the central trajectory. On the other end, the energy compensation by the RF cavity is accompanied by a momentum increase that is directed along the longitudinal direction of the central trajectory, parallel to the cavity axis. Though the energy exchange is perfectly balanced, the exchange of the particle momentum components causes a reduction of the amplitude of the transverse (horizontal and vertical) motion. Let us introduce then Cooling in the equations above.

Eq. (19) for the betatron emittance of the beam is now replacd by

$$
\mathrm{d} \varepsilon / \mathrm{dt}=-\alpha_{\mathrm{T}} \varepsilon+\mathrm{D}_{\mathrm{T}}
$$

where the diffusion rate $\mathrm{D}_{\mathrm{T}}$ is given by Eq. (19) itself, and the damping rate

$$
\alpha_{\mathrm{T}} \quad=\quad(\mathrm{dE} / \mathrm{dt}) / \beta p c \mathrm{~A}
$$

The equilibrium emittance $\varepsilon_{\infty}=\mathrm{D}_{\mathrm{T}} / \alpha_{\mathrm{T}}$ is reached after a time $\mathrm{T}_{\mathrm{T}} \sim 3 / \alpha_{\mathrm{T}}$. The results for the three cases are shown in Table 3. Shown are also the number of revolutions $n_{\mathrm{T}}$ corresponding to $\mathrm{T}_{\mathrm{T}}$, and the actual beam half size (horizontal and vertical) expected for $\beta_{\mathrm{L}}=1 \mathrm{~m}$. Thus Ionization Cooling of the betatron motion is very beneficial in all cases.

TABLE 3. Ionization Cooling.

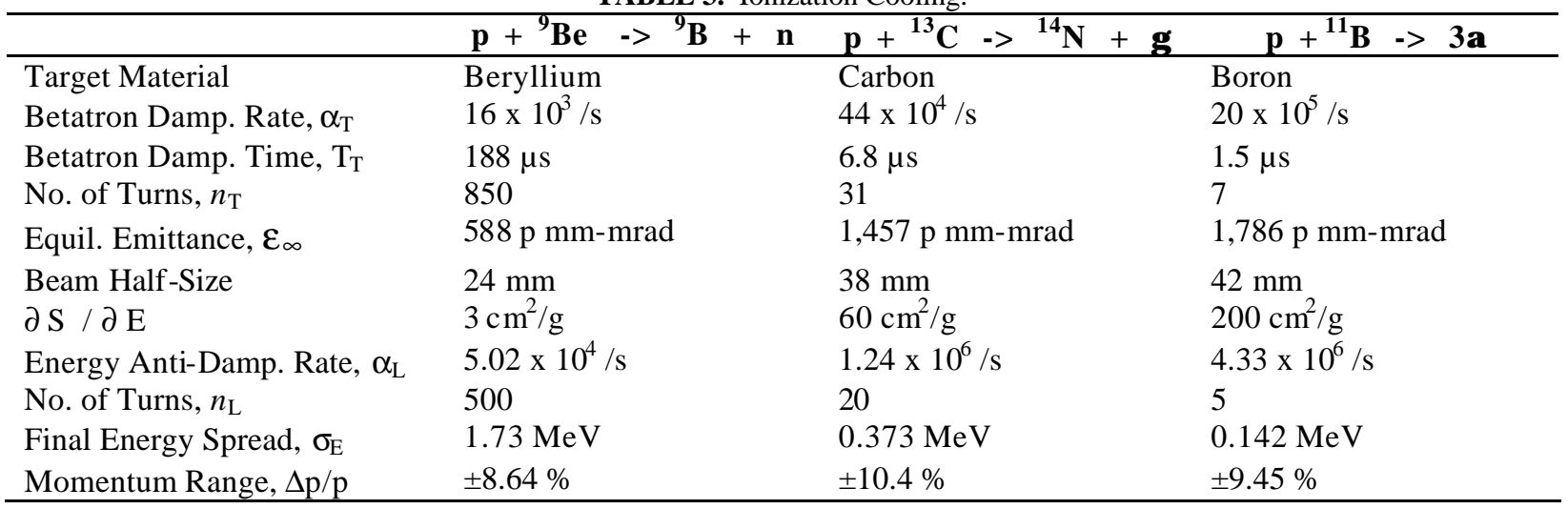

Unfortunately the situation is not as good in the longitudinal direction of motion where now the equation for the rms energy spread becomes

$$
\mathrm{d} \sigma_{\mathrm{E}}^{2} / \mathrm{dt} \quad=\quad \alpha_{\mathrm{L}}{\sigma_{\mathrm{E}}^{2}}^{2} \quad \mathrm{D}_{\mathrm{L}}
$$

where $\mathrm{D}_{\mathrm{L}}=\mathrm{d} \sigma_{\mathrm{E}}^{2} / \mathrm{dt}$ is the diffusion rate from scattering with the electrons in the Foil given by Eq.(22) and listed in Table 2 , and $\alpha_{\mathrm{L}}$ is the anti-damping rate

$$
\alpha_{\mathrm{L}} \quad=\quad 2(g / \mathrm{C}) \beta c \delta \partial \mathrm{S} / \partial \mathrm{E}
$$

The derivative $\partial \mathrm{S} / \partial \mathrm{E}$ can be derived from the same Tables from which the stopping power $\mathrm{S}$ was derived [2]. It is also listed in Table 3 for the three cases under consideration. Because the primary beam energy is low in all cases, the derivative $\partial \mathrm{S} / \partial \mathrm{E}$ is positive, that is anti-damping. The situation would be inverted for sufficiently larger energy $(\sim 2 \mathrm{GeV})$, in which case there is damping also of the beam energy spread. 
Assuming that at $t=0 \quad \sigma_{\mathrm{E}}=0$ or very small, the integration of the diffusion Eq. (26) gives

$$
\sigma_{\mathrm{E}}^{2}=\left(\mathrm{D}_{\mathrm{L}} / \alpha_{\mathrm{L}}\right)\left[\exp \left(\alpha_{\mathrm{L}} t\right)-1\right]
$$

Thus the energy spread will grow exponentially. To limit the amount of growth, one should let the beam circulate only for a short period $t=n_{\mathrm{L}} / f_{0}$ before it is disposed. The results are shown in Table 3 and they correspond to a Foil thickness of $10 \mu \mathrm{m}$. It is of course possible to lengthen the number $n_{\mathrm{L}}$ of circulation turns by correspondingly reducing the thickness of the Foil $g$. For instance, a thickness of Beryllium Foil of $5 \mu \mathrm{m}$ would allow 1,000 revolutions, and a Boron thickness of $1 \mu \mathrm{m}$ would allow the more comfortable number of 50 revolutions.

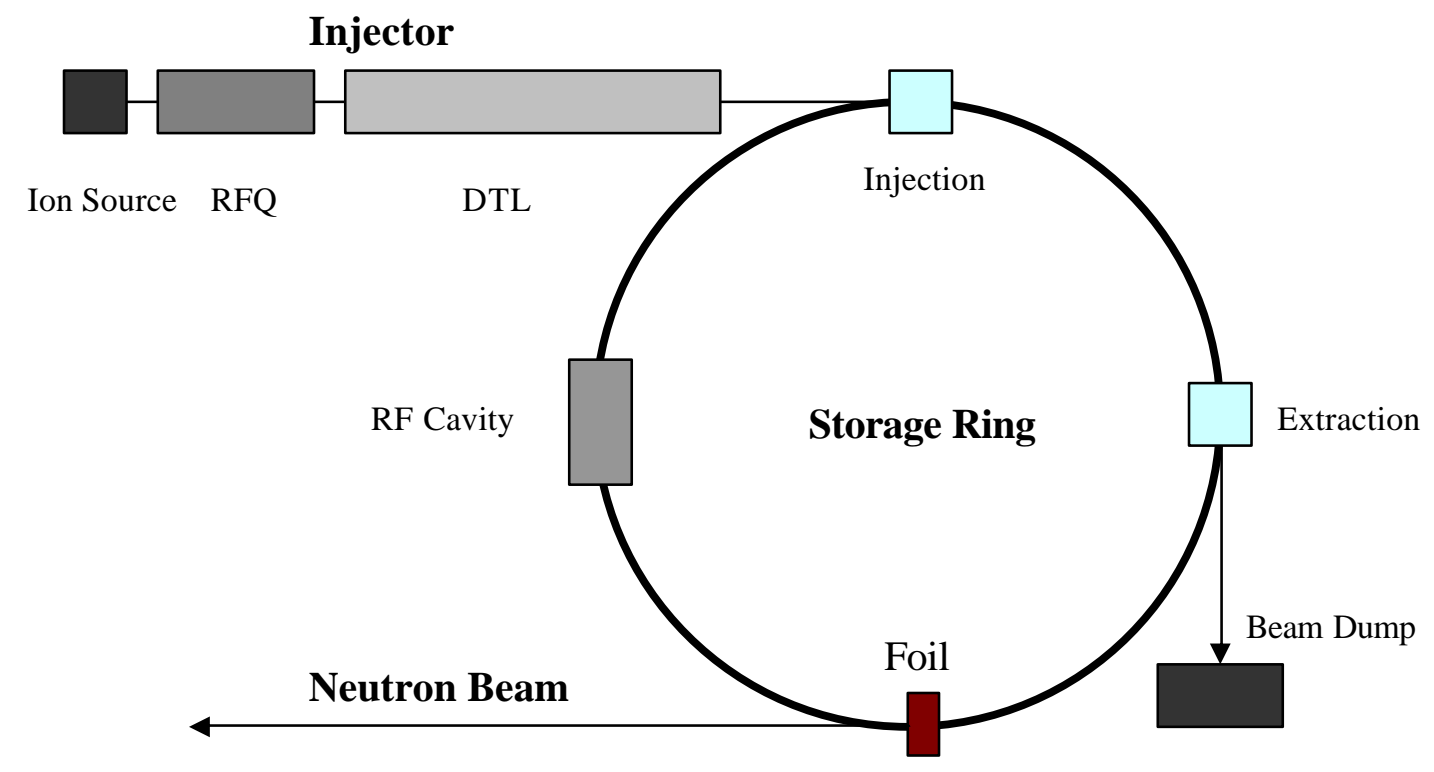

Figure 1. Secondary Beam Production based on the ERIT Principle

\section{THE MERIT OF THE ERIT SCHEME}

The experimental layout we have described here is made of two major components: a Linear Accelerator as the Injector that provides the primary beam at full energy, and the Storage Ring where the beam circulates for $n_{\mathrm{L}}$ revolutions before being disposed. Injection and extraction are done in a single turn, and thus are fast and take only a little time much shorter than the storage time $t=n_{\mathrm{L}} / f_{0}$. The Storage Ring does not provide further acceleration but only the re-use of the same beam for the continuous production of secondary particles.

In principle there may be no need of the Storage Ring, since the primary beam in exit of the Linear Accelerator can directly be transported to the Foil in a Single Pass mode. In this mode though a large number of particles would be wasted, and the beam power is large. For instance, for the production of $10^{12}$ neutrons per second, one needs a continuous beam current on the Foil of $7.2 \mathrm{~mA}$ and a kinetic energy of $10 \mathrm{MeV}$, corresponding to a continuous beam power of $72 \mathrm{~kW}$ for the Single-Pass mode. But if the beam circulates 1,000 revolutions in the Storage Ring the average beam power is reduced by the same factor down to 72 Watts. Thus, for any of the applications considered above, the inverse of the number $n_{\mathrm{L}}$ of revolutions in the Storage Ring is a measure of the Multiple-Pass mode duty cycle: the larger $n_{\mathrm{L}}$ correspondingly lower is the average beam power.

The Storage Ring must have the capability to store a beam with a large energy spread. It has been proposed that it can be done with a Fixed-Field Alternating-Gradient (FFAG) Storage Ring. In this case the relevant parameter is the final momentum spread $\Delta \mathrm{p} / \mathrm{p}$ of the beam at the end of the storage period, listed at the bottom of Table 3 . The required momentum acceptance for such Storage Ring is then about $\pm 10 \%$ for all cases. 


\section{REFERENCES}

1. Y. Mori, "Secondary Particle Source with FFAG-ERIT Scheme", Proceedings of the International Workshop on FFAG Accelerators, KURRI, Osaka, Japan, Dec. 5-9, 2005. Page 15.

2. American Institute of Physics Handbook. McGraw-Hill Book Company. Third Edition. Section 8d: Passage of Charged Particles through Matter. Pages 8-142 to 8-189.

3. D. Neuffer, "Ionization Cooling in a Low-Energy Proton Storage Ring", Proceedings of the International Workshop on FFAG Accelerators, KURRI, Osaka, Japan, Dec. 5-9, 2005. Page 21. 